\title{
CPT Violation and the Nature of Neutrinos
}

\author{
G. Barenboim ${ }^{a}$, J.F. Beacom ${ }^{b}$, L. Borissov $^{c}$, B. Kayser $^{\mathrm{a}}$ \\ ${ }^{a}$ Theoretical Physics Department, Fermi National Accelerator Laboratory, Batavia, \\ Illinois 60510-0500, USA \\ ${ }^{\mathrm{b}}$ NASA/Fermilab Astrophysics Center, Fermi National Accelerator Laboratory, \\ Batavia, Illinois 60510-0500, USA \\ ${ }^{\mathrm{c}}$ Physics Department, Columbia University, New York, New York 10027, USA
}

\begin{abstract}
In order to accommodate the neutrino oscillation signals from the solar, atmospheric, and LSND data, a sterile fourth neutrino is generally invoked, though the fits to the data are becoming more and more constrained. However, it has recently been shown that the data can be explained with only three neutrinos, if one invokes CPT violation to allow different masses and mixing angles for neutrinos and antineutrinos. We explore the nature of neutrinos in such CPT-violating scenarios. Majorana neutrino masses are allowed, but in general, there are no longer Majorana neutrinos in the conventional sense. However, CPT-violating models still have interesting consequences for neutrinoless double beta decay. Compared to the usual case, while the larger mass scale (from LSND) may appear, a greater degree of suppression can also occur.
\end{abstract}

Key words: Neutrino mass and mixing, double beta decay

PACS: 14.60.Pq, 23.40.-s

FERMILAB-Pub-02/014-T, FERMILAB-Pub-02/014-A

\section{Introduction}

In recent years, stronger and stronger experimental evidence for neutrino oscillations has been accumulating. As is well-known, this evidence would extend the Standard Model by requiring neutrino masses and mixings. While knowing the values of the mass and mixing parameters may be an important clue to physics beyond the Standard Model, more information is needed. For example,

Email addresses: gabriela@fnal.gov (G. Barenboim), beacom@fnal.gov (J.F. Beacom), liubo@phys.columbia.edu (L. Borissov), boris@fnal.gov (B. Kayser).

Preprint submitted to Elsevier Science 9 November 2018 
it is presently unknown whether neutrino processes violate lepton number or not, or whether the neutrinos are their own antiparticles or not.

Neutrino masses and mixings can be straightforwardly included in the Standard Model, as can the choice of Majorana or Dirac neutrinos. But in fact, the present neutrino oscillation evidence may imply more profound extensions to the Standard Model. With three neutrinos, there are only two independent mass-squared differences. However, since the mass-squared differences required by the solar[1], atmospheric [2], and LSND [3] neutrinos are $\delta m^{2} \simeq 10^{-5}$ $\mathrm{eV}^{2}, 10^{-3} \mathrm{eV}^{2}$, and $1 \mathrm{eV}^{2}$, respectively, three different mass-squared scales are needed to explain all the data. The usual way out is to postulate a fourth neutrino, thus allowing three independent mass-squared differences. This fourth neutrino must be sterile under the weak interactions since the invisible width of the $Z^{\circ}$ only allows three light active neutrinos.

The LSND results are based on an appearance experiment, namely $\bar{\nu}_{\mu} \rightarrow \bar{\nu}_{e}$. For years, the solar and atmospheric neutrino results only gave clear evidence for the disappearance of $\nu_{e}$ or $\nu_{\mu}, \bar{\nu}_{\mu}$, respectively. Thus one or the other could have been accommodated by oscillations to sterile neutrinos. However, the difficulties that seem to require the introduction of a fourth neutrino have recently become more acute as the combined solar neutrino results from the Sudbury Neutrino Observatory and Super-Kamiokande indicate that $\nu_{e} \rightarrow$ $\nu_{\mu}, \nu_{\tau}$, and the atmospheric neutrino results from Super-Kamiokande indicate that $\nu_{\mu}, \bar{\nu}_{\mu} \rightarrow \nu_{\tau}, \bar{\nu}_{\tau}$. Thus with three signals of neutrino oscillations among active flavors, there is not only a problem of not enough independent masssquared differences, but also a problem of where to incorporate the required mixing with the sterile neutrino. While four-neutrino models may still work, it is only with difficulty (see, e.g., Ref. [4]).

Recently, an intriguing but speculative suggestion to accommodate these results has been made [5,6] (see also Ref. [7]). If CPT is violated in the neutrino sector, then the mixing parameters which govern solar neutrino oscillations of $\nu_{e} \rightarrow \nu_{\mu}, \nu_{\tau}$ do not have to be the same as those that govern $\bar{\nu}_{\mu} \rightarrow \bar{\nu}_{e}$ in LSND. Without introducing new particles or interactions to the Standard Model, this allows the introduction of a new mass-squared difference and it also solves the problem of which flavors are oscillating to which. In the simplest model, atmospheric neutrino oscillations of $\nu_{\mu} \rightarrow \nu_{\tau}$ behave the same as their antiparticles.

Thus, it is not merely that the Standard Model must be extended to accomodate neutrino mass and mixing. With the present data, taking all of the experimental results at face value, one can either introduce new particles (sterile neutrinos), or allow CPT violation. Either suggestion, if confirmed, would be an important clue for understanding physics well beyond the Standard Model. Large CPT violation in the neutrino sector, while a radical suggestion, 
should be easily testable in the upcoming experiments.

Previous papers have explored the consequences of large CPT violation in the neutrino sector [5-13]. Here we explore the consequences for the Majorana or Dirac nature of the neutrinos (for an introduction in the CPT-conserving case, see Ref. [14]). For CPT-conserving neutrinos, it is frequently stated that "If neutrinoless double beta decay is observed, then neutrinos are Majorana particles," meaning that a neutrino and its antineutrino are the same state. We show that for CPT-violating neutrinos, a more careful statement must be made, i.e., "If neutrinoless double beta decay is observed, then there must be Majorana neutrino mass terms," and that in general, neutrinos are not equal to the corresponding antineutrinos as states. In either the CPT-conserving or CPT-violating case, the observation of neutrinoless double beta decay would of course imply the violation of lepton number. Needless to say, if one can determine the nature of the neutrino masses, light will be shed on the symmetries of fundamental particle interactions.

We also show that there are interesting practical consequences for neutrinoless double beta decay, a process that violates lepton number by two units. One way to explain the present null' $\square$ results [17] in the usual CPT-conserving scenario would be to say that the neutrino masses are all below say, $0.1 \mathrm{eV}$ (larger masses can be accommodated if the mixing angles and Majorana phases cause cancellations). Given present constraints on the oscillation parameters, there can be a non-zero minimum allowed effective mass. In the CPT-violating model the overall scale may be set by the large LSND mass scale of $\sim 1 \mathrm{eV}$, i.e., predicting a larger signal. However, a larger degree of suppression can occur, so that the effective mass can also be arbitrarily small.

\section{CPT Violation and the Neutrino Masses}

In the Standard Model, extended to include neutrino masses, the interactions are lepton-number (L) conserving. Any nonconservation of L would come from Majorana mass terms, which turn a neutrino into an antineutrino. When CPT is conserved and Majorana mass terms are present, the neutrino mass eigenstates $\nu_{i}$ are Majorana particles. That is, each $\nu_{i}$ is its own antiparticle in the sense that

$$
C P T\left|\nu_{i}\right\rangle=e^{i \xi_{i}}\left|\nu_{i}\right\rangle
$$

$\overline{1}$ Though evidence for neutrinoless double beta decay has very recently been claimed in Ref. [15], it has been disputed in Refs. [10,16]. 
where $\xi_{i}$ is a phase. Through the process pictured in Fig. 1, exchange of the Majorana $\nu_{i}$ leads to neutrinoless double beta decay $\left(\beta \beta_{0 \nu}\right)$. This is the Lnonconserving reaction in which one nucleus decays to another with the emission of two electrons. Conversely, even if $\beta \beta_{0 \nu}$ should arise predominantly from some mechanism other than light neutrino exchange, the observation of this decay would imply that the electron neutrino $\nu_{e}$ has a nonzero Majorana mass. When CPT is conserved, this would in turn imply that the mass eigenstates $\nu_{i}$ are Majorana particles. In a CPT-conserving world, when there are no Majorana mass terms, $\mathrm{L}$ is conserved, $\beta \beta_{0 \nu}$ is forbidden, and the mass eigenstates $\nu_{i}$ are Dirac particles. That is, each $\nu_{i}$ differs from its CPT conjugate by the value of the conserved quantum number $\mathrm{L}: \nu_{i}$ has $\mathrm{L}=+1$ while $\bar{\nu}_{i}$ has $\mathrm{L}=-1$.

To see what becomes of this picture when CPT is violated, we consider the simplest case of a single neutrino $\nu$ (i.e., $\nu_{e}$ ), coupled to the electron by the Standard Model weak coupling, and its CPT conjugate $\bar{\nu}$, coupled to the positron. When allowing CPT violation, we do not assume that $\nu$ and $\bar{\nu}$ have the same mass. Rather, we suppose that for a given spin direction, the $\nu, \bar{\nu}$ mass matrix $M_{\nu}$ has the form

$$
M_{\nu}=\left[\begin{array}{cc}
\mu+\Delta & y^{*} \\
y & \mu-\Delta
\end{array}\right]
$$

where the first and second rows correspond to $\nu$ and $\bar{\nu}$, respectively. We neglect the possibility of neutrino decay, so that $M_{\nu}$ must be Hermitian, which implies that the mass parameters $\mu$ and $\Delta$ (Dirac masses) are real. Any nonvanishing $\Delta$ is a violation of the CPT constraint that a particle and its antiparticle must have the same mass. Any nonvanishing $y$ (Majorana mass), which mixes $\nu$ and $\bar{\nu}$, is a violation of $\mathrm{L}$ conservation. (Note that limits on CPT violation entering via a heavy Majorana mass have been considered in Ref. [13]).

The eigenstates of $M_{\nu}$ are

$$
\left|\nu_{+}\right\rangle=\cos \theta_{\nu \bar{\nu}}|\nu\rangle+e^{i \phi} \sin \theta_{\nu \bar{\nu}}|\bar{\nu}\rangle
$$

with mass $m_{+}=\mu+\sqrt{|y|^{2}+\Delta^{2}}$, and

$$
\left|\nu_{-}\right\rangle=-\sin \theta_{\nu \bar{\nu}}|\nu\rangle+e^{i \phi} \cos \theta_{\nu \bar{\nu}}|\bar{\nu}\rangle
$$

with mass $m_{-}=\mu-\sqrt{|y|^{2}+\Delta^{2}}$. The neutrino-antineutrino mixing angle is given by

$$
\tan 2 \theta_{\nu \bar{\nu}}=\frac{|y|}{\Delta}
$$


and $\phi=\arg (y)$.

Under CPT, $\nu$ and $\bar{\nu}$ transform as

$$
C P T|\nu\rangle=e^{i \xi}|\bar{\nu}\rangle, C P T|\bar{\nu}\rangle=e^{i \xi}|\nu\rangle
$$

where we have left the phase $\xi$ free. Since CPT is an antiunitary operator,

$$
C P T(C P T|\nu\rangle)=C P T\left(e^{i \xi}|\bar{\nu}\rangle\right)=e^{-i \xi} C P T|\bar{\nu}\rangle=e^{i(\xi-\xi)}|\nu\rangle=|\nu\rangle .
$$

From Eqs. $(3,4)$,

$$
C P T\left|\nu_{+}\right\rangle=e^{i(\xi-\phi)}\left[\sin \theta_{\nu \bar{\nu}}|\nu\rangle+e^{i \phi} \cos \theta_{\nu \bar{\nu}}|\bar{\nu}\rangle\right]
$$

and

$$
C P T\left|\nu_{-}\right\rangle=-e^{i(\xi-\phi)}\left[-\cos \theta_{\nu \bar{\nu}}|\nu\rangle+e^{i \phi} \sin \theta_{\nu \bar{\nu}}|\bar{\nu}\rangle\right]
$$

Comparing with Eqs. $(3,4)$, one sees that the mass eigenstates $\nu_{ \pm}$are Majorana states (that is, CPT self-conjugate apart from a phase) if and only if $\theta_{\nu \bar{\nu}}=\pi / 4$. But, from Eq. (5), this value of $\theta_{\nu \bar{\nu}}$ corresponds to $\Delta=0$; i.e., to an absence of CPT violation. If $\Delta \neq 0$, so that CPT is not conserved, the neutrino mass eigenstates can no longer be Majorana particles. Nevertheless, if $y$ is nonzero, then there is $\nu-\bar{\nu}$ mixing, $\mathrm{L}$ is not conserved, and $\beta \beta_{0 \nu}$ can occur. But if it does occur, that would imply only that $\mathrm{L}$ is violated and that there is a "Majorana" (i.e., $\nu-\bar{\nu}$ mixing) mass term, and not that the neutrino mass eigenstates are CPT self-conjugate.

It is interesting to compare this situation with the neutral kaon system when a possible CPT-violating term is introduced as a difference between the $K^{\circ}$ and $\bar{K}^{\circ}$ masses (e.g., see Ref. [18]). To compare to neutrinos, we neglect kaon decay. Then the kaon mass matrix is identical to the $M_{\nu}$ of Eq. (2), but with the first and second rows now corresponding to $K^{\circ}$ and $\bar{K}^{\circ}$, respectively. The neutral kaon mass eigenstates (these correspond to $K_{S}$ and $K_{L}$ in the usual case) are described by Eqs. (3) and (4), with $\nu$ replaced by $K^{\circ}$ and $\bar{\nu}$ replaced by $\bar{K}^{\circ}$. The CPT conjugates of these states are described by Eqs. (8) and (9), with the same replacements. Once again, the mass eigenstates are CPT selfconjugate if and only if the CPT-violating parameter $\Delta$ vanishes. Otherwise, each of them differs from its CPT conjugate. Nevertheless, so long as the "Majorana" $K^{\circ}-\bar{K}^{\circ}$ mixing term $y$ is present, then strangeness (playing the role of $\mathrm{L}$ ) is not conserved. A kaon born as a $K^{\circ}$ can evolve into a $K^{\circ}-\bar{K}^{\circ}$ mixture. Observing, via the scattering of the kaon, that such evolution had occurred, would imply that strangeness is not conserved, and in particular, that $K^{\circ}$ and $\bar{K}^{\circ}$ mix, but not that the mass eigenstates are self-conjugate. 


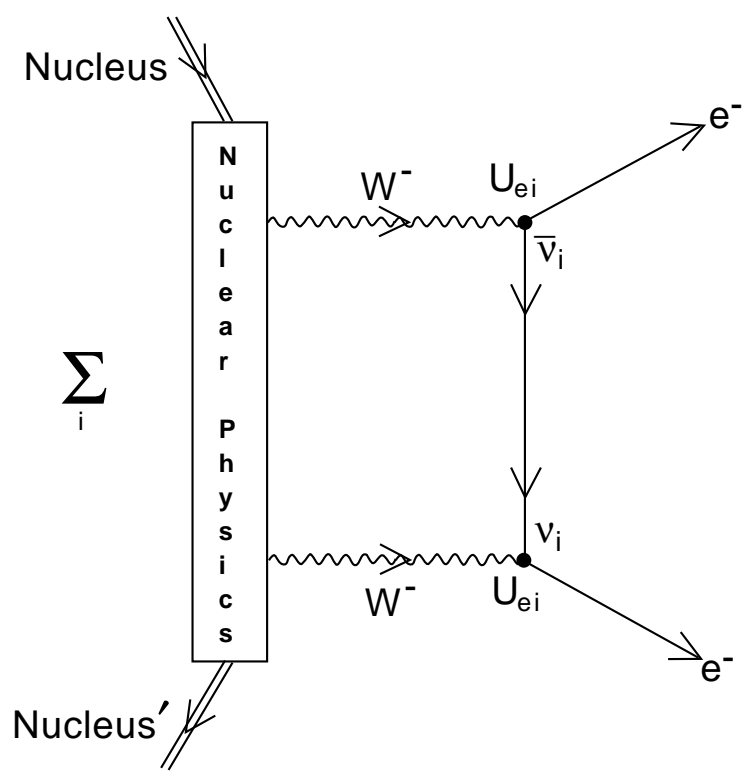

Fig. 1. Diagram for neutrinoless double beta decay and the leptonic amplitude considered below. This diagram corresponds to the CPT-conserving case; the modifications for the CPT-violating case are considered below.

\section{Neutrinoless Double Beta Decay}

Neutrinoless double beta decay [17] is the process

$$
(A, Z) \longrightarrow(A, Z+2)+2 e^{-}
$$

in which a nucleus decays into another nucleus with the emission of two electron; see Fig. 1. The emission of two $W$ bosons by the initial nucleus is described by a nuclear matrix element, not considered here. The full amplitude for this process is proportional to just the leptonic amplitude, which is

$$
A=\sum_{i, h}\left\langle e^{-} e^{-}\left|H_{W}\right| e^{-} \nu_{i} W^{-}\right\rangle\left\langle e^{-} \nu_{i} W^{-}\left|H_{W}\right| W^{-} W^{-}\right\rangle
$$

where $i$ labels the neutrino mass eigenstates and $h$ their helicities. Each mass eigenstate can be represented as a superposition of neutrinos and antineutrinos, so that

$$
\left|\nu_{i}\right\rangle=\sum_{\alpha=e, \mu, \tau} U_{\alpha i}\left|\nu_{\alpha}\right\rangle+\sum_{\alpha=e, \mu, \tau} \bar{U}_{\alpha i}^{*}\left|\bar{\nu}_{\alpha}\right\rangle
$$

which defines the matrices $U$ and $\bar{U}$ for neutrinos and antineutrinos, respectively. Absorbing a $\nu_{l}$ yields an $l^{-}$, and similarly for a $\bar{\nu}_{l}$ and an $l^{+}$. Because of 
the helicity mismatch between the neutrino and antineutrino interaction, the leptonic amplitude is proportional to

$$
A \propto \sum_{i} m_{i} U_{e i} \bar{U}_{e i}
$$

where the sum is over mass eigenstates. In particular, for the one-flavor case discussed above,

$$
A \propto \sin \theta_{\nu \bar{\nu}} \cos \theta_{\nu \bar{\nu}}\left(m_{+}-m_{-}\right)=\sin 2 \theta_{\nu \bar{\nu}} \sqrt{|y|^{2}+\Delta^{2}}=|y|
$$

As expected, the Majorana mass term $y$ contributes to the amplitude. In fact, with just a single neutrino flavor, it is the only contribution, i.e., the value of $\Delta$ is irrelevant. In the more relevant case where more than one neutrino family is involved, there are in general contributions depending on the CPT-violating mass term $\Delta$, and hence the LSND scale of $1 \mathrm{eV}$. We show this with an explicit example below. Even at this stage, it is easy to understand that if the neutrino and antineutrino mass matrices are different, the diagonalizing matrices must also be different. The difference will be reflected in the matrices $U$ and $\bar{U}$ in Eq. (13). The degree of difference depends on $\Delta$, the CPT-violating mass term, and of course vanishes when $\Delta=0$.

Note that the usual CPT-conserving amplitude is obtained with $\bar{U}=U$ in Eq. (13). At this point two remarks are in order. First, one should remember that in a CPT-violating world $\bar{U} \neq U$ and second, Eq. (13) is obtained without depending on the usual Feynman rules by constructing the amplitudes from quantum mechanics and therefore is quite safe regardless whether our neutrinos conserve CPT or not. It must be emphasized that the same expression can also be obtained by using the method suggested in [6], i.e., by calculating the matrix elements as if they belonged to a CPT-conserving neutrino in an artificial background of matter. In this case (as it happens in field theory at finite temperature) the Feynman rules for the physical vertices are the same as those in the CPT-conserving theory. The propagators, however, are different. For momenta much higher than the neutrino masses, the propagator in matter can be written as an expansion in powers of the CPT violating mass term, whose first term is the standard propagator.

Double beta decay will have no manifest CPT violation, in the sense that the CPT-conjugated process involving two positrons will have exactly the same rate. This observation follows from the fact that the corresponding amplitude for two positrons is simply the complex conjugate of that for two electrons. However, CPT violation does have an experimentally observable consequence. Namely, since in the CPT-violating case the double beta decay amplitude involves two independent matrices $U$ and $\bar{U}$, the decay rate can reach values that are outside those allowed in the usual CPT-conserving case. 
In order to make this statement even more transparent, we first recall the result for the CPT-conserving case. The usual expression (assuming only two flavors and degenerate masses) is

$$
m_{\beta \beta}=m_{0}\left|\cos ^{2} \theta+\sin ^{2} \theta e^{2 i \alpha}\right|
$$

where $\theta$ is the angle involved in the solar neutrino solution and $\alpha$ is a $\mathrm{CP}$ violating phase characteristic of Majorana neutrinos which does not appear in oscillation-related phenomena. Since SNO sees $1 / 3$ of the expected $\nu_{e}$ flux (i.e., less than $1 / 2$ ), the mixing angle must be less than maximal (the best-fit value is about $30^{\circ}$ ) [1]. Thus there is a minimum value for $m_{\beta \beta}$, obtained when $\alpha=\pi / 2$. The range for $m_{\beta \beta}$ is thus

$$
m_{0} \cos \left(2 \theta_{\odot}\right)<m_{\beta \beta}<m_{0}
$$

Presently, $\cos \left(2 \theta_{\odot}\right) \simeq 0.4$, and the overall mass scale can be very small. In the more general three-flavor CPT-conserving case, the expression for $m_{\beta \beta}$ is more complicated, but it retains the feature of a minimum value (except at a singular point) [19].

When CPT is violated, the neutrino and antineutrino mixing matrices can be different, and so can the neutrino and antineutrino masses. If MiniBooNE confirms LSND, then we know that a mass of about $1 \mathrm{eV}$ exists (this is the case of most interest to CPT violation). This mass scale is larger than required in the absence of the LSND oscillation signal, raising hope that it might give a large neutrinoless double beta decay signal. In general, it is much more complicated to treat the full problem, where there are now six mass eigenstates, each containing components of both neutrinos and antineutrinos of all three flavors. This can be visualized by merging the two spectra in Fig. (1) in Ref. [5], and allowing neutrino-antineutrino admixtures, permitted by the Majorana masses. That combined spectrum is consistent with the solar, atmospheric, and LSND data.

However, we know that for this spectrum the state with mass corresponding to the LSND mass scale is the dominant term in the sum for $m_{\beta \beta}$ given by Eq. (13), which is then reduced to

$$
m_{\beta \beta} \simeq m_{L S N D} U_{e, L S N D} \bar{U}_{e, L S N D}
$$

In this picture, $\left|U_{e, L S N D}\right|^{2}$ represents the electron neutrino content of the highest mass eigenstate, and similarly for $\left|\bar{U}_{e, L S N D}\right|^{2}$ and the electron antineutrino content. In order to explain all the data, the electron antineutrino content should be very large $(\simeq 99 \%)[6]$, and the remainder can be filled by other neutrino and antineutrino flavors. These proportions in particular can satisfy 
the bounds on reactor $\bar{\nu}_{e}$ disappearance. In the case where this remainder is mostly the electron neutrino component, then $m_{\beta \beta}$ reaches it maximal value, given by $\simeq 0.1 m_{L S N D}$. On the other hand, there is no lower limit of the electron neutrino content, so finally,

$$
0<m_{\beta \beta}<0.1 m_{L S N D}
$$

Though beyond the scope of this work, it would be very interesting to consider in more detail the allowed numerical range of $m_{\beta \beta}$ in realistic three-flavor models with CPT violation.

\section{Conclusions}

Present and forthcoming experiments devoted to studying neutrino oscillations should give us much more information about the neutrino mass-squared differences and mixing angles. However, in order to really understand the neutrino sector we will also need to know the absolute mass scale and the nature (Dirac or Majorana) of the neutrino mass terms. Neutrinoless double beta decay experiments are crucial in this respect, as well as for studying lepton flavor violation.

We have explored the nature of neutrinos when CPT is violated. Contrary to the widespread belief that CPT-violating neutrinos can have only a Dirac character and therefore no neutrinoless beta decay can be expected, we have shown that CPT violation can also be seen in neutrinoless double beta decay experiments. As an important general point, though Majorana neutrino masses are allowed, in general, there are no longer Majorana neutrino states in the conventional sense. If the CPT-violating neutrino mixing model is chosen to explain the LSND result, then at least one mass is of order the large LSND scale of $1 \mathrm{eV}$. This can increase the neutrinoless double beta decay rate relative to the usual case in which all neutrino masses can be small. On the other hand, due to the freedom in the mixing between the neutrino and antineutrino in each mass eigenstate, a greater degree of suppression in the effective mass that appears in neutrinoless beta decay is also possible.

\section{Acknowledgements}

We thank J. Lykken for discussions. G.B., J.F.B (as the David N. Schramm Fellow), and B.K. were supported by Fermilab, which is operated by URA under DOE contract No. DE-AC02-76CH03000. J.F.B. was also supported by NASA under NAG5-10842. L.B. was supported by the Sloan Foundation. 


\section{References}

[1] B. T. Cleveland et al. [Homestake Collaboration], Astrophys. J. 496, 505 (1998); W. Hampel et al. [GALLEX Collaboration], Phys. Lett. B 447, 127 (1999); J. N. Abdurashitov et al. [SAGE Collaboration], Phys. Rev. C 60, 055801 (1999);

S. Fukuda et al. [SuperKamiokande Collaboration], Phys. Rev. Lett. 86, 5651 (2001); ibid, 5656 (2001);

M. Altmann et al. [GNO Collaboration], Phys. Lett. B 490, 16 (2000);

Q. R. Ahmad et al. [SNO Collaboration], Phys. Rev. Lett. 87, 071301 (2001).

[2] S. Fukuda et al. [Super-Kamiokande Collaboration], Phys. Rev. Lett. 85, 3999 (2000); Y. Fukuda et al., ibid, 81, 1562 (1998).

[3] A. Aguilar et al. [LSND Collaboration], Phys. Rev. D 64, 112007 (2001).

[4] M. C. Gonzalez-Garcia and Y. Nir, hep-ph/0202058.

[5] G. Barenboim, L. Borissov, J. Lykken and A. Y. Smirnov, hep-ph/0108199.

[6] G. Barenboim, L. Borissov and J. Lykken, hep-ph/0201080.

[7] H. Murayama and T. Yanagida, Phys. Lett. B 520, 263 (2001).

[8] S. Skadhauge, hep-ph/0112189.

[9] S. M. Bilenky, M. Freund, M. Lindner, T. Ohlsson and W. Winter, hep$\mathrm{ph} / 0112226$.

[10] A. Strumia, hep-ph/0201134.

[11] J. N. Bahcall, V. Barger and D. Marfatia, hep-ph/0201211.

[12] O. W. Greenberg, hep-ph/0201258.

[13] I. Mocioiu and M. Pospelov, hep-ph/0202160.

[14] B. Kayser, "The Physics of Massive Neutrinos," (World Scientific, Singapore, 1989).

[15] H. V. Klapdor-Kleingrothaus, A. Dietz, H. L. Harney and I. V. Krivosheina, Mod. Phys. Lett. A 16, 2409 (2002).

[16] C. E. Aalseth et al., hep-ex/0202018.

[17] S. R. Elliott and P. Vogel, hep-ph/0202264;

P. Vogel, in "Current Aspects of Neutrino Physics," ed. D.O. Caldwell (Springer-Verlag, Berlin, 2001), nucl-th/0005020;

D. E. Groom et al., Eur. Phys. J. C 15, 1 (2000).

[18] C. O. Dib and R. D. Peccei, Phys. Rev. D 46, 2265 (1992).

[19] F. Vissani, JHEP 9906, 022 (1999);

S. Pascoli, S. T. Petcov and L. Wolfenstein, Phys. Lett. B 524, 319 (2002). 